\title{
On the Stability Problem of Stationary Solutions for the Euler Equation on a 2-Dimensional Torus
}

\author{
P. Buttà $\quad$ P. Negrini ${ }^{\dagger}$ \\ Dipartimento di Matematica, SAPIENZA Università di Roma, \\ P.le Aldo Moro 2, 00185 Roma, Italy
}

\begin{abstract}
We study the linear stability problem of the stationary solution $\psi^{*}=$ $-\cos y$ for the Euler equation on a 2-dimensional flat torus of sides $2 \pi L$ and $2 \pi$. We show that $\psi^{*}$ is stable if $L \in(0,1)$ and that exponentially unstable modes occur in a right neighborhood of $L=n$ for any integer $n$. As a corollary, we gain exponentially instability for any $L$ large enough and an unbounded growth of the number of unstable modes as $L$ diverges.
\end{abstract}

MSC2010 numbers: 76E05, 35Q35, 34B08

Key words: Euler equation, shear flows, linear stability

\section{Introduction}

Given $L>0$, let $\mathbb{T}_{L}^{2}$ be the 2-dimensional torus of sides $2 \pi L$ and $2 \pi$,

$$
\mathbb{T}_{L}^{2}:=\{(x, y): x \in \mathbb{R} / 2 \pi L \mathbb{Z}, \quad y \in \mathbb{R} / 2 \pi \mathbb{Z}\} .
$$

We consider the Euler equation on $\mathbb{T}_{L}^{2}$, written in terms of the stream function $\psi \in C^{1}\left(\mathbb{R}_{+} ; W^{2,2}\left(\mathbb{T}_{L}^{2} ; \mathbb{R}\right)\right)$,

$$
\frac{\partial \Delta \psi}{\partial t}+\langle J \nabla \psi, \nabla \Delta \psi\rangle=0
$$

with $J$ the antisymmetric matrix

$$
J=\left(\begin{array}{cc}
0 & 1 \\
-1 & 0
\end{array}\right)
$$

As it is well known, this equation admits the following two independent first integrals,

$$
I_{1}:=\int_{\mathbb{T}_{L}^{2}} \mathrm{~d} x \mathrm{~d} y|\nabla \psi|^{2}, \quad I_{2}:=\int_{\mathbb{T}_{L}^{2}} \mathrm{~d} x \mathrm{~d} y(\Delta \psi)^{2} .
$$

*E-mail: butta@mat.uniroma1.it

${ }^{\dagger}$ E-mail: piero.negrini@uniroma1.it 
Any regular function on $\mathbb{T}_{L}^{2}$ depending only on the $y$ or on the $x$ variable is a stationary solution to (1.1). More generally, any function $\phi$ such that $\Delta \phi$ is functionally dependent on $\phi$ is a stationary solution. This is obviously verified by the eigenfunctions of the Laplacian. In particular, in this paper we are interested in the stability problem of the stationary solution $\psi^{*}:=-\cos y$. This old problem was firstly posed by Arnol'd, see Example 2 in [2, Appendix 2] or Example 4.6 in [3]. More specifically, the question is to analyze the stability of the stationary solution $\psi^{*}$ as $L$ crosses the critical value $L=1$.

We rewrite (1.1) in terms of the shifted stream function $\Psi:=\psi+\cos y$,

$$
\frac{\partial \Delta \Psi}{\partial t}+\sin y \frac{\partial}{\partial x}(\Psi+\Delta \Psi)+\langle J \nabla \Psi, \nabla \Delta \Psi\rangle=0 .
$$

By disregarding the nonlinear terms in (1.3) we obtain the linear equation,

$$
\frac{\partial \Delta \Psi}{\partial t}+\sin y \frac{\partial}{\partial x}(\Psi+\Delta \Psi)=0
$$

According to Arnol'd, the stationary solution $\Psi^{*}=0$ is unstable [resp. stable] for $L>1$ [resp. $0<L \leq 1$ ], and the proof of instability can be achieved by means of continued fractions techniques, firstly applied by Meshalkin and Sinai in [7] in the case of the Navier-Stokes flow. Previous announcement of this result was given in the report [4] by Arnol'd and Meshalkin.

Few years after the Meshalkin-Sinai paper, V.I. Yudovich [8] studied with the same techniques the bifurcation phenomenon occurring when there is a loss of stability of the laminar flow of a viscous incompressible fluid. In recent years, following Arnol'd suggestion of using continued fractions, Friedlander at al. [6] studied the linear instability of the stationary solution $\psi^{(m)}=-\frac{1}{m} \cos m y$, $m \in \mathbb{N}$, to (1.1). The instability property of $\psi^{(m)}$ relies on the existence of roots with positive real part of an infinite algebraic system of complex variable, with coefficients depending on $L$. Each root with positive real part determines the exponential growth of an unstable mode. In the particular case $m=1$, real roots exist for $L>1$. Moreover, numerical calculations indicate that there exists exactly one real root for each $L^{1}$. More general results for $m>1$ can be found in the quoted papers.

We begin Section 2 by briefly discussing the problem of the linear stability of $\psi^{*}$ for $L \leq 1$. The main part of the section is devoted to give a new proof of the exponential instability of $\psi^{*}$ when $L>1$. Our strategy relies on proving the existence of $2 \pi$-periodic solutions to a second order non autonomous linear differential equation (the Raleigh equation), whose coefficients depend on two parameters $(\omega, \varepsilon) \in \mathbb{C} \times \mathbb{R}$. We translate the problem into a functional system which is solved by a perturbative approach in the cone $\mathcal{K}:=\left\{\omega \in \mathbb{C}: 0<\Re \omega<\frac{1}{2}, \quad|\Im \omega| \leq|\Re \omega|\right\}$, obtaining a unique solution $\omega=\omega(\varepsilon)$ defined in a right neighborhood of $\varepsilon=0$. This function ensures

\footnotetext{
${ }^{1}$ Indeed, in [7] it is proven that in the case of non zero viscosity the analogous equation has only real roots.
} 
the existence of periodic solutions for the corresponding one parameter differential equation. Moreover, $\Re \omega(\varepsilon)$ gives the rate of the exponential growth of the unstable modes of the linearized equation (1.4). In our opinion, beyond the instability result, this perturbative analysis is of some interest by itself.

To conclude, we notice that $\Re \omega(\varepsilon)=O(\varepsilon), \Im \omega(\varepsilon)=o(\varepsilon)$. We are not able to prove that $\Im \omega(\varepsilon) \equiv 0$, but the numerical result in [6] strongly support this conclusion.

\section{The linearized equation}

Quite obviously, Eq. (1.4) admits the first integrals

$$
\mathcal{I}:=\frac{1}{(2 \pi)^{2} L} \int_{\mathbb{T}_{L}^{2}} \mathrm{~d} x \mathrm{~d} y\left[(\Delta \Psi)^{2}-|\nabla \Psi|^{2}\right]
$$

and

$$
\mathcal{I}_{k_{2}}:=\int_{\mathbb{T}_{L}^{2}} \mathrm{~d} x \mathrm{~d} y \mathrm{e}^{\mathrm{i} k_{2} y} \Delta \Psi, \quad k_{2} \in \mathbb{Z} .
$$

We emphasize that, for any $L \leq 1$, the first integral $\mathcal{I}$ is a non-negative but not positive-definite functional. Therefore, also the linear stability for $L \leq 1$ is not a straightforward property. Writing $\Psi$ in Fourier expansion,

$$
\Psi(x, y)=\sum_{k \in \mathbb{Z}^{2}} \Psi_{k_{1}, k_{2}} \mathrm{e}^{\mathrm{i}\left(\frac{k_{1}}{L} x+k_{2} y\right)},
$$

Eq. (1.4) translates into the following infinite system of O.D.E. for the modes $\Psi_{k_{1}, k_{2}}$,

$$
\begin{aligned}
{\left[\left(\frac{k_{1}}{L}\right)^{2}+k_{2}^{2}\right] \dot{\Psi}_{k_{1}, k_{2}}=} & \frac{k_{1}}{2 L}\left\{\left[\left(\frac{k_{1}}{L}\right)^{2}+k_{2}^{2}+2 k_{2}\right] \Psi_{k_{1}, k_{2}+1}\right. \\
& \left.-\left[\left(\frac{k_{1}}{L}\right)^{2}+k_{2}^{2}-2 k_{2}\right] \Psi_{k_{1}, k_{2}-1}\right\} .
\end{aligned}
$$

Notice that system (2.3) splits into an infinite set of independent subsystems $\mathcal{S}_{k_{1}}, k_{1} \in \mathbb{Z}$. The integrals $\mathcal{I}$ and $\mathcal{I}_{k_{2}}$, written in terms of the Fourier coefficients, read

$$
\begin{gathered}
\mathcal{I}=\sum_{\left(k_{1}, k_{2}\right) \in \mathbb{Z}^{2}}\left[\left(\frac{k_{1}}{L}\right)^{2}+k_{2}^{2}\right]\left[\left(\frac{k_{1}}{L}\right)^{2}+k_{2}^{2}-1\right]\left|\Psi_{k_{1}, k_{2}}\right|^{2}, \\
\mathcal{I}_{k_{2}}=-k_{2}^{2} \Psi_{0, k_{2}}, \quad k_{2} \in \mathbb{Z} .
\end{gathered}
$$

The first integral (2.4) is given by the sum $\sum_{k_{1} \in \mathbb{Z}} \mathcal{I}_{k_{1}}$ of the following first integrals for the subsystems $\mathcal{S}_{k_{1}}$,

$$
\mathcal{I}_{k_{1}}:=\sum_{k_{2} \in \mathbb{Z}}\left[\left(\frac{k_{1}}{L}\right)^{2}+k_{2}^{2}\right]\left[\left(\frac{k_{1}}{L}\right)^{2}+k_{2}^{2}-1\right]\left|\Psi_{k_{1}, k_{2}}\right|^{2}, \quad k_{1} \in \mathbb{Z} .
$$


Each subsystem $\mathcal{S}_{k_{1}}$ also admits two further first integrals (possibly formal),

$$
\begin{gathered}
\mathcal{H}_{k_{1}}^{+}:=\sum_{k_{2} \in \mathbb{Z}}\left[\left(\frac{k_{1}}{L}\right)^{2}+k_{2}^{2}\right] \Psi_{k_{1}, k_{2}}, \quad k_{1} \in \mathbb{Z}, \\
\mathcal{H}_{k_{1}}^{-}:=\sum_{k_{2} \in \mathbb{Z}}(-1)^{k_{2}}\left[\left(\frac{k_{1}}{L}\right)^{2}+k_{2}^{2}\right] \Psi_{k_{1}, k_{2}}, \quad k_{1} \in \mathbb{Z},
\end{gathered}
$$

which are obtained by evaluating the linear equation (1.4) at $y=0$ and $y=\pi$.

We now discuss the stability problem of the zero solution to (1.4) for different values of the parameter $L$.

Theorem 2.1 If $0<L<1$ the zero solution to (1.4) is stable.

Proof. By (2.4), the conservation of $\mathcal{I}$ guarantees that all the Fourier coefficients $\Psi_{k_{1}, k_{2}}$ are controlled except the coefficient $\Psi_{0,1}$, which is, however, a first integral, see (2.5).

Remark 2.2 Of course, this result does not imply the stability of the zero solution for the nonlinear equation (1.3), because $\mathcal{I}_{k_{2}}$ is not conserved by this evolution.

The stability properties in the case $L=1$ are still an open problem for the linear equation (1.4) and, a fortiori, for the nonlinear equation (1.3). Here we only give an idea on the difficulties for the linear case. Integral (2.6) does not give a control on the mode $\Psi_{1,0}$ and we are left with the study of the subsystem $\mathcal{S}_{1}$, which is given now by the equation

$$
\dot{\Psi}_{1,0}=\frac{1}{2}\left(\Psi_{1,1}-\Psi_{1,-1}\right),
$$

coupled with the two subsystems $\mathcal{S}_{1}^{ \pm}$defined by

$$
\left(1+k_{2}^{2}\right) \dot{\Psi}_{1, k_{2}}=\frac{1}{2}\left[\left(k_{2}+1\right)^{2} \Psi_{1, k_{2}+1}-\left(k_{2}-1\right)^{2} \Psi_{1, k_{2}-1}\right],
$$

with $k_{2} \geq 1$ for $\mathcal{S}_{1}^{+}$and $k_{2} \leq-1$ for $\mathcal{S}_{1}^{-}$respectively. We note that $\mathcal{S}_{1}^{ \pm}$admit the first integrals

$$
\mathcal{I}_{1}^{ \pm}=\sum_{k_{2} \in \mathbb{N}} k_{2}^{2}\left(1+k_{2}^{2}\right)\left|\Psi_{1, \pm k_{2}}\right|^{2},
$$

which give a control on the sequences $\left\{a_{k_{2}}^{ \pm} ; k_{2} \in \mathbb{N}\right\}, a_{k_{2}}^{ \pm}=k_{2}^{2}\left|\Psi_{1, \pm k_{2}}\right|$, in $\ell_{2}$. However, the first integrals $\mathcal{H}_{1}^{ \pm}$defined in (2.7)-(2.8), which could control also the mode $\Psi_{1,0}$, are finite only if $\left\{a_{k_{2}}^{ \pm} ; k_{2} \in \mathbb{N}\right\}$ are sequences in $\ell_{1}$.

Now, we consider the case $L>1$.

Theorem 2.3 The zero solution of Eq. (1.4) is exponentially unstable for $L$ belonging to a right neighborhood of $L=1$. Moreover, there exists a number $L_{0} \geq 1$ such that the zero solution is unstable for all $L>L_{0}$. 
Theorem 2.3 is a straightforward consequence of the following main theorem.

Theorem 2.4 The set $\mathbb{N}$ of positive integers is a bifurcation set. More precisely, there exist $\varepsilon_{0}>0$, functions $\omega^{ \pm} \in C^{1}\left(\left[0, \varepsilon_{0}\right) ; \mathbb{C}\right)$ with $\Re \omega^{ \pm}(\varepsilon)>0$ for all $\varepsilon \in$ $\left(0, \varepsilon_{0}\right)$, and a solution to (1.4) of the form

$$
\Psi^{ \pm}(x, y, t)=\mathrm{e}^{\frac{k}{L_{k}(\varepsilon)}\left(\mathrm{i} x \pm \omega^{ \pm}(\varepsilon) t\right)} f^{ \pm}(y, \varepsilon)
$$

where $f^{ \pm}(\cdot, \varepsilon) \in C^{2}\left(S^{1} ; \mathbb{C}\right)$ and $L_{k}(\varepsilon):=k(1+\varepsilon), k \in \mathbb{N}$.

Remark 2.5 We emphasize that by Theorem 2.4 the number of unstable modes for the zero solution is diverging as $L \rightarrow \infty$. Note in fact that, letting $n=n(L)$ be the largest positive integer such that $\frac{L}{[L]-n}<1+\varepsilon_{0}$, we have $n(L) \rightarrow \infty$ as $L \rightarrow \infty$, and for each $k=[L]-n, \ldots,[L]$ there exists $\varepsilon_{k} \in\left(0, \varepsilon_{0}\right)$ such that $k\left(1+\varepsilon_{k}\right)=L$. There are therefore $n+1$ unstable modes. This growth of unstable modes is the analogous to that one for the Navier-Stokes equation. Indeed, the dimension of the unstable manifold of the Kolmogorov flow is equal to $2[L]$, see $[5]$.

Proof of Theorem 2.4. We consider the case of unstable modes. Given $\varepsilon \in(0,1)$ and $\omega \in \mathbb{C}$, a function of the form

$$
\Psi(x, y, t)=\mathrm{e}^{\frac{1}{1+\varepsilon}(\mathrm{i} x+\omega t)} f(y)
$$

is a solution to (1.4) if and only if $f(y)$ is a $2 \pi$-periodic solution of the ordinary differential equation,

$$
\frac{\mathrm{d}^{2} f}{\mathrm{~d} y^{2}}-\frac{\mathrm{i} \omega+\varepsilon(2+\varepsilon) \sin y}{(1+\varepsilon)^{2}(\mathrm{i} \omega-\sin y)} f=0 .
$$

Our strategy is to prove, by a perturbative method, that this solution does exist at least for small $\varepsilon$ and $\omega$. It is not restrictive to assume $f(0)=1$. Let $f(y, \omega, \beta, \varepsilon), \beta \in \mathbb{C}$, be the solution to $(2.9)$ with $f(0)=1$ and $f^{\prime}(0)=\beta$. Clearly $f(y, \omega, \beta, \varepsilon)$ solves the integral equation,

$$
f(y)=1+\beta y+\int_{0}^{y} \mathrm{~d} \tau(y-\tau) \frac{\mathrm{i} \omega+\varepsilon(2+\varepsilon) \sin \tau}{(1+\varepsilon)^{2}(\mathrm{i} \omega-\sin \tau)} f(\tau) .
$$

By imposing periodic boundary conditions we obtain the following system of equations in the variables $(\omega, \beta, \varepsilon)$,

$$
\left\{\begin{array}{l}
\int_{0}^{2 \pi} \mathrm{d} \tau G(\tau, \omega, \varepsilon) f(\tau, \omega, \beta, \varepsilon)=0, \\
\int_{0}^{2 \pi} \mathrm{d} \tau \tau G(\tau, \omega, \varepsilon) f(\tau, \omega, \beta, \varepsilon)=\beta,
\end{array}\right.
$$

where

$$
G(\tau, \omega, \varepsilon):=\frac{i \omega+\varepsilon(2+\varepsilon) \sin \tau}{2 \pi(1+\varepsilon)^{2}(i \omega-\sin \tau)}
$$


Our purpose is to solve system (2.11) with respect to $(\omega, \beta)$, getting functions $\omega=\omega^{+}(\varepsilon), \beta=\beta(\varepsilon)$ (defined in a right neighborhood of $\varepsilon=0$ ). Setting

$$
R(y, \omega, \beta, \varepsilon):=f(y, \omega, \beta, \varepsilon)-1-\beta y,
$$

we rewrite (2.11) as follows,

$$
\left\{\begin{array}{l}
\mathcal{E}_{0}(\omega, \varepsilon)+\mathcal{E}_{1}(\omega, \varepsilon) \beta+A_{0}(\omega, \beta, \varepsilon)=0, \\
\mathcal{E}_{1}(\omega, \varepsilon)+\mathcal{E}_{2}(\omega, \varepsilon) \beta+A_{1}(\omega, \beta, \varepsilon)=\beta,
\end{array}\right.
$$

where

$$
\begin{gathered}
\mathcal{E}_{n}(\omega, \varepsilon)=\int_{0}^{2 \pi} \mathrm{d} \tau \tau^{n} G(\tau, \omega, \varepsilon), \quad n=0,1,2, \\
A_{n}(\omega, \beta, \varepsilon)=\int_{0}^{2 \pi} \mathrm{d} \tau \tau^{n} G(\tau, \omega, \varepsilon) R(\tau, \omega, \beta, \varepsilon), \quad n=0,1 .
\end{gathered}
$$

We notice that

$$
\mathcal{E}_{0}(\omega, 0)=\frac{\mathrm{i} \omega}{2 \pi} \int_{0}^{2 \pi} \frac{\mathrm{d} \tau}{\mathrm{i} \omega-\sin \tau}=0 \quad \text { if } \Re \omega=0 \text { and }|\Im \omega|<1 .
$$

Due to this degeneracy in $(2.14)_{1}$, the standard implicit function theorem does not apply. To overcome this difficulty we instead exploit a fixed point argument in a suitable cone. Let $\mathcal{K}:=\{\omega \in \mathbb{C}: \Re \omega>0,|\Im \omega| \leq|\Re \omega|\}$ and define $\mathcal{K}_{1 / 2}:=\left\{\omega \in \mathcal{K}:|\omega| \leq \frac{1}{2}\right\}$. The following two lemmata will be proved in the Appendix.

Lemma 2.6 For any $\omega \in \mathcal{K}_{1 / 2}$,

$$
\begin{gathered}
\mathcal{E}_{0}(\omega, \varepsilon)=\omega-2 \varepsilon+O_{2}(\omega, \varepsilon), \\
\mathcal{E}_{1}(\omega, \varepsilon)=i \omega \log \omega^{-1}+O_{1}(\omega, \varepsilon), \\
\mathcal{E}_{2}(\omega, \varepsilon)=2 \pi i \omega \log \omega^{-1}+O_{1}(\omega, \varepsilon),
\end{gathered}
$$

where $O_{k}(\omega, \varepsilon)$ denotes a regular function of order $k$ in $\mathcal{K}_{1 / 2} \times \mathbb{R}_{+}$.

Lemma 2.7 The functions $A_{n}(\omega, \beta, \varepsilon), n=0,1$, are continuously differentiable in $\mathcal{K} \times \mathbb{C} \times[0,1]$. Moreover, there exists a constant $C_{0}>0$ such that, for any $(\omega, \beta) \in \mathcal{K}_{1 / 2} \times \mathbb{C}$ and $\varepsilon \in[0,1]$,

$$
\begin{gathered}
\left|A_{n}(\omega, \beta, \varepsilon)\right| \leq C_{0}(1+|\beta|)\left(\varepsilon+|\omega| \log |\omega|^{-1}\right)^{2} \\
\left|\frac{\partial A_{n}}{\partial \omega}(\omega, \beta, \varepsilon)\right| \leq C_{0}(1+|\beta|)\left(\varepsilon+|\omega| \log |\omega|^{-1}\right) \log |\omega|^{-1} \\
\left|\frac{\partial A_{n}}{\partial \beta}(\omega, \beta, \varepsilon)\right| \leq C_{0}\left(\varepsilon+|\omega| \log |\omega|^{-1}\right)^{2}
\end{gathered}
$$


Coming back to sistem (2.14), by plugging the second equation into the first one, we can rewrite the latter in the form

$$
\omega=T_{\beta, \varepsilon}(\omega),
$$

where

$$
\begin{aligned}
T_{\beta, \varepsilon}(\omega):= & \omega-\mathcal{E}_{0}(\omega, \varepsilon)-\mathcal{E}_{1}(\omega, \varepsilon)^{2}-\mathcal{E}_{1}(\omega, \varepsilon) \mathcal{E}_{2}(\omega, \varepsilon) \beta \\
& -\mathcal{E}_{1}(\omega, \varepsilon) A_{1}(\omega, \beta, \varepsilon)-A_{0}(\omega, \beta, \varepsilon) .
\end{aligned}
$$

By the above lemmata, there exists a constant $\bar{C}>0$ such that, for any $\omega \in$ $\mathcal{K}_{1 / 2},|\beta| \leq 1$, and $\varepsilon \in[0,1]$,

$$
\begin{gathered}
\left|\Re T_{\beta, \varepsilon}(\omega)-2 \varepsilon\right| \leq \bar{C}\left(\varepsilon+|\omega| \log |\omega|^{-1}\right)^{2}, \\
\left|\Im T_{\beta, \varepsilon}(\omega)\right| \leq \bar{C}\left(\varepsilon+|\omega| \log |\omega|^{-1}\right)^{2}, \\
\left|\frac{\partial T_{\beta, \varepsilon}(\omega)}{\partial \omega}\right| \leq \bar{C}\left(\varepsilon+|\omega| \log |\omega|^{-1}\right) \log |\omega|^{-1}, \\
\left|\frac{\partial T_{\beta, \varepsilon}(\omega)}{\partial \beta}\right| \leq \bar{C}\left(\varepsilon+|\omega| \log |\omega|^{-1}\right)^{2} .
\end{gathered}
$$

By inequalities (2.18), (2.19), and (2.20), there exists $\varepsilon_{0}^{\prime}>0$ such that, for any $|\beta| \leq 1$ and $\varepsilon \in\left(0, \varepsilon_{0}^{\prime}\right)$, the compact set $S_{\varepsilon}:=\{\omega \in \mathcal{K}: \varepsilon \leq \Re \omega \leq 3 \varepsilon\}$ is invariant under the map $T_{\beta, \varepsilon}$ and $T_{\beta, \varepsilon}$ is a contraction on $S_{\varepsilon}$. Therefore, the fixed point equation (2.16) can be solved for any $|\beta| \leq 1$ and $\varepsilon \in\left(0, \varepsilon_{0}^{\prime}\right)$, getting $\omega=\bar{\omega}(\beta, \varepsilon)$ with $\varepsilon \leq \Re \bar{\omega}(\beta, \varepsilon) \leq 3 \varepsilon$. Moreover, by $(2.20)$ and $(2.21)$, for some constant $C^{*}>0$,

$$
\left|\frac{\partial \bar{\omega}}{\partial \beta}(\beta, \varepsilon)\right| \leq C^{*}\left(\varepsilon \log \varepsilon^{-1}\right)^{2} .
$$

Plugging $\omega=\bar{\omega}(\beta, \varepsilon)$ into the second equation of system (2.14), the latter assume the form

$$
\beta=Q_{\varepsilon}(\beta) .
$$

By the above lemmata and (2.22), there exists $\varepsilon_{0} \in\left(0, \varepsilon_{0}^{\prime}\right]$ such that, for any $\varepsilon \in\left(0, \varepsilon_{0}\right)$ the unit disk $\{\beta \in \mathbb{C}:|\beta| \leq 1\}$ is invariant under the map $Q_{\varepsilon}$ and $Q_{\varepsilon}$ is a contraction on it. Therefore, the fixed point equation (2.23) can be solved for $\varepsilon \in\left(0, \varepsilon_{0}\right)$, getting $\beta=\beta(\varepsilon)$. The claim of the theorem now follows with $\omega^{+}(\varepsilon):=\bar{\omega}(\beta(\varepsilon), \varepsilon)$. The proof for the stable modes is identical, by working in the negative cone.

\section{Appendix}

Proof of Lemma 2.6. We have

$$
\mathcal{E}_{n}(\omega, \varepsilon)=-\frac{\omega}{2 \pi \mathrm{i}} I_{n}(\omega)-\frac{(2 \pi)^{n} \varepsilon(2+\varepsilon)}{(n+1)(1+\varepsilon)^{2}},
$$


where

$$
I_{n}(\omega)=\int_{0}^{2 \pi} \mathrm{d} \tau \frac{\tau^{n}}{\mathrm{i} \omega-\sin \tau}
$$

Therefore, it is sufficient to evaluate $I_{n}(\omega)$ for $|\omega| \leq \frac{1}{2},|\Im \omega| \leq|\Re \omega|$ and $\Re \omega>0$. Under this assumption, there exists $K_{0}>0$ such that the function $F(z)=$ $z^{n}(\mathrm{i} \omega-\sin z)^{-1}$ has a unique pole $z_{0}$ in the complex domain

$$
D_{K}:=\{z \in \mathbb{C}: \Re z \in[0,2 \pi], \Im z \in[-K, 0]\},
$$

for any $K>K_{0}$. Moreover

$$
z_{0}=\pi+\Im \omega-\mathrm{i} \Re \omega+O_{3}(\omega) .
$$

Therefore, denoting with $\Gamma_{K}$ the boundary of $D_{K}$, by the Residue Theorem we have

$$
\frac{1}{2 \pi \mathrm{i}} \oint_{\Gamma_{K}} \mathrm{~d} z \frac{z^{n}}{\mathrm{i} \omega-\sin z}=-\frac{z_{0}^{n}}{\cos z_{0}}=\pi^{n}+O_{1}(\omega) \quad \forall K>K_{0} .
$$

On the other hand,

$$
\lim _{K \rightarrow \infty} \frac{1}{2 \pi \mathrm{i}} \oint_{\Gamma_{K}} \mathrm{~d} z \frac{z^{n}}{\mathrm{i} \omega-\sin z}=-\frac{1}{2 \pi \mathrm{i}} I_{n}(\omega)+C_{n}(\omega),
$$

with

$$
C_{n}(\omega)= \begin{cases}0 & \text { if } n=0, \\ -\mathrm{i} \int_{0}^{\infty} \mathrm{d} \sigma \frac{1}{\omega+\sinh \sigma} & \text { if } n=1, \\ -2 \pi \mathrm{i} \int_{0}^{\infty} \mathrm{d} \sigma \frac{1}{\omega+\sinh \sigma}-2 \int_{0}^{\infty} \mathrm{d} \sigma \frac{\sigma}{\omega+\sinh \sigma} & \text { if } n=2,\end{cases}
$$

where

$$
\int_{0}^{\infty} \mathrm{d} \sigma \frac{1}{\omega+\sinh \sigma}=\frac{1}{\sqrt{1+\omega^{2}}} \log \frac{\left(1+\omega+\sqrt{1+\omega^{2}}\right)^{2}}{2 \omega}
$$

and

$$
\sup _{\omega \in \mathcal{K}_{1 / 2}}\left|\int_{0}^{\infty} \mathrm{d} \sigma \frac{\sigma}{\omega+\sinh \sigma}\right|<\infty, \quad \sup _{\omega \in \mathcal{K}_{1 / 2}}\left|\omega \frac{\partial}{\partial \omega}\left[\int_{0}^{\infty} \mathrm{d} \sigma \frac{\sigma}{\omega+\sinh \sigma}\right]\right|<\infty .
$$

The claim of the lemma follows immediately from the previous computations.

Proof of Lemma 2.7. We remark that the solution to (2.10) is regular with respect to the parameters $(\omega, \beta, \varepsilon)$ for $\omega \in \mathcal{K} \backslash\{0\}$, the singularities being located 
at $(y, \omega)=(0,0)$ and $(y, \omega)=(\pi, 0)$. Recalling $(2.12)$, the function $R(y, \omega, \beta, \varepsilon)$ defined in (2.13) satisfies the integral equation,

$$
R(y, \omega, \beta, \varepsilon)=Q(y, \omega, \beta, \varepsilon)+2 \pi \int_{0}^{y} \mathrm{~d} \tau(y-\tau) G(\tau, \omega, \varepsilon) R(\tau, \omega, \beta, \varepsilon),
$$

where

$$
Q(y, \omega, \beta, \varepsilon)=2 \pi \int_{0}^{y} \mathrm{~d} \tau(y-\tau)(1+\beta \tau) G(\tau, \omega, \varepsilon) .
$$

For any $y \in[0,2 \pi],(\omega, \beta) \in \mathcal{K}_{1 / 2} \times \mathbb{C}$, and $\varepsilon \in[0,1]$, we have,

$$
\begin{aligned}
&|Q(y, \omega, \beta, \varepsilon)| \leq 2 \pi(1+2 \pi|\beta|) \int_{0}^{2 \pi} \mathrm{d} \tau\left[\frac{\varepsilon(2+\varepsilon)}{(1+\varepsilon)^{2}}+\frac{|\omega|}{|\mathrm{i} \omega-\sin \tau|}\right] \\
& \leq 2 \pi(1+2 \pi|\beta|) \int_{0}^{2 \pi} \mathrm{d} \tau\left[\varepsilon+\frac{2 \Re \omega}{\sqrt{(\Re \omega)^{2}+(\Im \omega+\sin \tau)^{2}}}\right] \\
& \leq C_{1}(1+|\beta|)\left(\varepsilon+|\omega| \log |\omega|^{-1}\right), \\
&|G(y, \omega, \varepsilon)| \leq \varepsilon+\frac{\Re \omega}{\pi \sqrt{(\Re \omega)^{2}+(\Im \omega+\sin y)^{2}}} \leq C_{1},
\end{aligned}
$$

where we used that $|\omega| \leq 2 \Re \omega \leq 2|\omega|$ for $\omega \in \mathcal{K}$, and $C_{1}$ is a suitable positive constant. Then, by the Gronwall lemma, for some $C_{2}>0$,

$$
|R(y, \omega, \beta, \varepsilon)| \leq C_{2}(1+|\beta|)\left(\varepsilon+|\omega| \log |\omega|^{-1}\right)
$$

for any $y \in[0,2 \pi],(\omega, \beta) \in \mathcal{K}_{1 / 2} \times \mathbb{C}$, and $\varepsilon \in[0,1]$.

Analogously, we next evaluate the derivatives of $R(y, \omega, \beta, \varepsilon)$ with respect to $(\omega, \beta)$. By (A.1),

$$
\begin{aligned}
& \frac{\partial R}{\partial \omega}(y, \omega, \beta, \varepsilon)=Q_{1}(y, \omega, \beta, \varepsilon)+2 \pi \int_{0}^{y} \mathrm{~d} \tau(y-\tau) G(\tau, \omega, \varepsilon) \frac{\partial R}{\partial \omega}(\tau, \omega, \beta, \varepsilon), \\
& \frac{\partial R}{\partial \beta}(y, \omega, \beta, \varepsilon)=Q_{2}(y, \omega, \beta, \varepsilon)+2 \pi \int_{0}^{y} \mathrm{~d} \tau(y-\tau) G(\tau, \omega, \varepsilon) \frac{\partial R}{\partial \beta}(\tau, \omega, \beta, \varepsilon),
\end{aligned}
$$

with

$$
\begin{gathered}
Q_{1}(y, \omega, \beta, \varepsilon)=2 \pi \int_{0}^{y} \mathrm{~d} \tau(y-\tau)[1+\beta \tau+R(\tau, \omega, \beta, \varepsilon)] \frac{\partial G}{\partial \omega}(\tau, \omega, \varepsilon), \\
Q_{2}(y, \omega, \beta, \varepsilon)=2 \pi \int_{0}^{y} \mathrm{~d} \tau(y-\tau) \tau G(\tau, \omega, \varepsilon) .
\end{gathered}
$$

We observe that, for any $y \in[0,2 \pi],(\omega, \beta) \in \mathcal{K}_{1 / 2} \times \mathbb{C}$, and $\varepsilon \in[0,1]$,

$$
\left|\frac{\partial G}{\partial \omega}(\tau, \omega, \varepsilon)\right| \leq \frac{1}{\pi \sqrt{(\Re \omega)^{2}+(\Im \omega+\sin y)^{2}}}+\frac{\Re \omega}{\pi\left[(\Re \omega)^{2}+(\Im \omega+\sin y)^{2}\right]} .
$$


Then, there exists $C_{3}>0$ such that, for any $y \in[0,2 \pi],(\omega, \beta) \in \mathcal{K}_{1 / 2} \times \mathbb{C}$, and $\varepsilon \in[0,1]$

$$
\begin{aligned}
&\left|Q_{1}(y, \omega, \beta, \varepsilon)\right| \leq C_{3}(1+|\beta|) \log |\omega|^{-1}, \\
&\left|Q_{2}(y, \omega, \beta, \varepsilon)\right| \leq C_{3}\left(\varepsilon+|\omega| \log |\omega|^{-1}\right),
\end{aligned}
$$

whence, by the Gronwall lemma, for some $C_{4}>0$,

$$
\begin{aligned}
&\left|\frac{\partial R}{\partial \omega}(y, \omega, \beta, \varepsilon)\right| \leq C_{4}(1+|\beta|) \log |\omega|^{-1}, \\
&\left|\frac{\partial R}{\partial \beta}(y, \omega, \beta, \varepsilon)\right| \leq C_{4}\left(\varepsilon+|\omega| \log |\omega|^{-1}\right) .
\end{aligned}
$$

The claim of the lemma now follows by the definition (2.15) and the estimates (A.2), (A.3), (A.4), (A.5), and (A.6).

\section{References}

[1] Arnol'd, V., Sur la géometrie différentielle des groupes de Lie de dimension infinie et ses applications à l'hydrodynamique des fluides parfaits, Ann. Inst. Fourier (Grenoble), 1966, vol. XXVI, pp. 319-361.

[2] Arnol'd, V., Mathematical Methods of Classical Mechanics, New York: Springer, 1977.

[3] Arnol'd, V., Topological Methods in Hydrodynamics, New York: Springer, 1998.

[4] Arnol'd, V. and Meshalkin L.D., A.N. Kolmogoroff's Seminar on selected problems of analysis (1958-1959), Uspekhi Mat. Nauk, vol. XV, (91). (English Translation: Russian Mathematical Surveys).

[5] Babin, A.V. and Vishik, A.A., Attractors of Evolution Equations, Amsterdam: North-Holland Publishing, 1992.

[6] Belenkaya, L., Friedlander, S.J., and Yudovich, V., The unstable spectrum of oscillating shear flows, Siam J. Appl. Math., vol. 59, (1999), pp. 17011715 .

[7] Meshalkin, L.D. and Sinai, Ya.G., Investigation of the stability of a stationary solution of a system of equations for the plane movement of an incompressible viscous liquid, Prikl. Mat. Meh., vol. 25, pp. 1140-1143 (in Russian); translated as J. Appl. Math. Mech., (1961), vol. 25, pp. 17001705.

[8] Yudovich, V., Example of the generation of a secondary stationary or periodic flow when there is a loss of stability of the laminar flow of a viscous incompressible fluid., Prikl. Mat. Meh., vol. 29, pp. 453-467 (in Russian); translated as J. Appl. Math. Mech., (1965), vol. 29, pp. 527-544. 